\title{
THE MARKUS-YAMABE CONJECTURE DOES NOT HOLD FOR PIECEWISE DISCONTINUOUS LINEAR DIFFERENTIAL SYSTEMS SEPARATED BY ONE STRAIGHT LINE
}

\author{
JAUME LLIBRE ${ }^{1}$, LUCYJANE DE A. S. MENEZES ${ }^{2}$
}

\begin{abstract}
The Markus-Yamabe conjecture is a conjecture on global asymptotic stability. The conjecture states that if a differentiable system $\dot{x}=f(x)$ has a singularity and the Jacobian matrix $D f(x)$ has everywhere eigenvalues with negative real part, then the singularity is a global attractor. In this paper we consider piecewise discontinuous linear differential systems in $\mathbb{R}^{2}$ separated by one straight line $\Sigma$ such that the unique singularity of the system is at $\Sigma$ and the Jacobian matrix of the system has everywhere eigenvalues with negative real part. We prove that these piecewise discontinuous linear differential systems can have one crossing limit cycle and consequently these systems do not satisfy the Markus-Yamabe conjecture.
\end{abstract}

\section{INTRODUCTION AND STATEMENT OF THE MAIN RESULTS}

Consider $f(x)$ a $C^{1}$ map on an $n$-dimensional real vector space. Let

$$
\dot{x}=f(x)
$$

be a differential system such that $f(0)=0$. In 1960 Markus and Yamabe stated that if all eigenvalues of $D f(x)$ have negative real part, then the origin of (1) is a global attractor. In their paper [8] the conjecture has been proved under some strong additional hypotheses. This statement became known as the Markus-Yamabe conjecture.

Many authors have dedicated in proving the Markus-Yamabe conjecture. The conjecture is true for $C^{1}$ two-dimensional systems. In 1988 Meisters and Olech proved the Markus-Yamabe conjecture for polynomial vector fields in the plane, see [9]. Considering vector fields of class $C^{1}$ defined in $\mathbb{R}^{2}$, Gutierrez [6], Fleber [3] and Glutsyuk [5] for this order provided different proofs of the Markus-Yamabe conjecture in the years 1994-5. However counterexamples

2010 Mathematics Subject Classification. Primary 34C05, 34C07, 37G15.

Key words and phrases. discontinuous differential system, limit cycle, Markus-Yamabe conjecture. 
have been constructed in higher dimension. Bernat and Llibre [1] in 1996 presented a counterexample to the conjecture in dimension larger than 3 . In the paper [2] of 1997, Cima et al. provided a counterexample for the case $n=3$. More precisely, they proved that the Markus-Yamabe conjecture is false for polynomial vector fields in $\mathbb{R}^{n}$ with $n \geq 3$.

We define a piecewise discontinuous linear Markus-Yamabe differential system, or simply piecewise Markus-Yamabe system as

$$
\dot{z}= \begin{cases}X(z) & \text { if } \quad x>0 \\ Y(z) & \text { if } \quad x<0\end{cases}
$$

where $z=(x, y) \in \mathbb{R}^{2}, X$ and $Y$ are linear vector fields, the real part of the eigenvalues of $D X(z)$ and $D Y(z)$ are negative, $Y(0)=0$, and the singularity of $X$ is virtual, i.e. it leaves in the half-plane $x<0$. The straight line $\Sigma=\left\{(x, y) \in \mathbb{R}^{2} ; x=0\right\}$ is called the discontinuity set. Here $\dot{z}$ denotes the derivative of $z$ with respect to the independent variable $t$, usually called the time.

In order to simplify the notation we denote the piecewise Markus-Yamabe system as $Z=(X, Y)$ and call it simply as piecewise $M Y$-system.

Observe that $X$ and $Y$ are linear differential systems whose singularities are of type foci $(\mathrm{F})$ or nodes $(\mathrm{N})$. Furthermore, the nodes can diagonalize with distinct eigenvalues $(N)$, or with equal eigenvalues $\left(N^{*}\right)$, or do not diagonalize $(i N)$. We say that $Z$ is a piecewise MY-system of type $L R$, with $L, R \in$ $\left\{F, N, N^{*}, i N\right\}$, when $Y$ has a singularity of type $L$ and $X$ has a singularity of type $R$.

The extension of the conjecture of Markus-Yamabe to piecewise MY-system claims: The origin of any piecewise $M Y$-system is a global attractor. Our main goal is to prove that this conjecture does not hold for piecewise MY-systems.

According to Filippov conventions [4] the discontinuity set $\Sigma$ of the piecewise MY-system is decomposed in escape, sliding and sewing regions. We define a crossing limit cycle as a limit cycle that is concatenation of two orbits one of the vector field $X$ and the other of the vector field $Y$ which connect in two sewing points. In what follows a crossing limit cycle will be called simply limit cycle. In order to see that the Markus-Yamabe conjecture does not hold for piecewise MY-system, we should characterize what piecewise MY-systems can have limit cycles. This characterization is done in the next result.

Theorem 1. A piecewise $M Y$-system of types FF, FN and FiN have at most one limit cycle, and there are systems of these types with exactly one 
limit cycle. The others piecewise $M Y$-systems different from these three types have no limit cycles.

The rest of the paper is organized as follows. In Section 2 we provide some basic notions and results that we shall need for proving Theorem 1 . In the short section 3 we prove the last part of Theorem 1, i.e. that the piecewise MYsystems of type $F N^{*}, N N^{*}, i N N$, and $i N N^{*}$ have no limit cycles. Finally in the long section 4 we prove the first part of Theorem 1, which we have divided it in three subsections one for each piecewise MY-system of type $F F, F N$ and FiN.

\section{Preliminary Results}

In this section we present the essential definitions and results that we need in this paper.

Consider $X$ and $Y$ linear vector fields and

$$
\dot{z}= \begin{cases}X(z) & \text { if } \quad x>0, \\ Y(z) & \text { if } \quad x<0,\end{cases}
$$

where $z=(x, y) \in \mathbb{R}^{2}$, a piecewise linear differential system whose discontinuity is the straight line $\Sigma=\left\{(x, y) \in \mathbb{R}^{2} ; x=0\right\}$.

We define the following singularities to system (3).

- Real singularity: $p=(x, y)$ such that $x>0$ and $X(p)=0$, or $x<0$ and $Y(p)=0$.

- Virtual singularity: $p=(x, y)$ such that $x>0$ and $Y(p)=0$, or $x<0$ and $X(p)=0$.

- Boundary singularity: $p \in \Sigma$ such that $X(p)=0$ or $Y(p)=0$.

We say that a point $(0, y)$ is an invisible fold point for the vector field $X=$ $\left(X_{1}, X_{2}\right)$ when

$$
X_{1}(0, y)=0 \quad \text { and } \quad \frac{\partial X_{1}}{\partial y}(0, y) X_{2}(0, y)<0
$$

and an invisible fold point for the vector field $Y=\left(Y_{1}, Y_{2}\right)$ when

$$
Y_{1}(0, y)=0 \quad \text { and } \quad \frac{\partial Y_{1}}{\partial y}(0, y) Y_{2}(0, y)>0 .
$$

A $T$-system is a linear differential system having a singularity of type $T$ with $T \in\left\{F, N, N^{*}, i N\right\}$. Next result provides a simpler way to rewrite a $T$-system in the plane and its proof can be found in Proposition 5 of [7]. 
Proposition 2. Let $M=\left(m_{i j}\right)$ be a $2 \times 2$ matrix. If the linear differential system

$$
(\dot{x}, \dot{y})^{T}=M(x, y)^{T}
$$

is a

(a) $N$ or $N^{*}$-system then after a vertical lines-preserving linear change of variables and a time-rescaling system (4) becomes $(\dot{x}, \dot{y})^{T}=M_{1}(x, y)^{T}$;

(b) F-system then after a vertical lines-preserving linear change of variables and a time-rescaling system (4) becomes $(\dot{x}, \dot{y})^{T}=M_{2}(x, y)^{T}$ with $a \neq 0$;

(c) iN-system then after a vertical lines-preserving linear change of variables and a time-rescaling system (4) becomes $(\dot{x}, \dot{y})^{T}=M_{3}(x, y)^{T}$,

where

$$
\begin{aligned}
& M_{1}=\left(\begin{array}{ll}
a & 1 \\
1 & a
\end{array}\right) \quad \text { with } \quad|a|>1 ; \quad M_{2}=\left(\begin{array}{cc}
a & 1 \\
-1 & a
\end{array}\right) \quad \text { with } a \in \mathbb{R} ; \\
& \text { and } M_{3}=\left(\begin{array}{ll}
\lambda & \lambda \\
0 & \lambda
\end{array}\right) \quad \text { with } \lambda= \pm 1 .
\end{aligned}
$$

The next result is the Lemma 6 of [7].

Lemma 3. We consider the functions

$$
\begin{aligned}
& F(t)=e^{-a t} \csc t-\cot t, \quad G(t)=e^{-a t} \operatorname{csch} t-\operatorname{coth} t, \\
& H(t)=\frac{e^{-t}-1}{t} .
\end{aligned}
$$

The following statements hold.

(a) For every $a<0$, the function $F(t)$ is monotonic increasing in the interval $(0, \pi)$ and $F(t)>-a$ for $t \in(0, \pi)$.

(b) For $a<-1$, the function $G(t)$ is monotonic increasing on $\mathbb{R}$ and $G(t)>$ $-a$ for $t>0$.

(c) The function $H(t)$ is monotonic increasing on $\mathbb{R}$ and $H(t)<-1$ for $t<0$.

\section{The MY-Systems of TYPe $F N^{*}, N N^{*}, i N N$ AND $i N N^{*}$}

Observe that, if the piecewise MY-system have one virtual singularity of type $N^{*}$, then the solution passing through the point $(0, y) \in \Sigma$ cannot return 
to $\Sigma$ because the eigenvalues of the Jacobian matrix are equal, that is the orbits of the vector field $Y$ leave in straight lines.

If the piecewise MY-system have one boundary singularity of type $N^{*}, N$ or $i N$, then the orbits of the vector field $X$ passing through $(0, y) \in \Sigma$ coincides with $\Sigma$ or returns to $\Sigma$ at the singularity. Therefore the first return map is not defined. Consequently the piecewise MY-system of type $F N^{*}, N N^{*}, i N N$ and $i N N^{*}$ do not have limit cycles. This proves the last part of Theorem 1 .

\section{Limit CyCles FOR PieCEWise MY-Systems}

Consider a piecewise discontinuous linear differential system defined in $\mathbb{R}^{2}$

$$
\dot{z}=\left\{\begin{array}{l}
X(z) \text { if } \quad x>0, \\
Y(z) \text { if } \quad x<0,
\end{array}\right.
$$

where $z=(x, y)$. We denote the solution of (6) by

$$
\varphi(t, \bar{x}, \bar{y})= \begin{cases}\varphi^{+}(t, \bar{x}, \bar{y}) & \text { if } \quad \bar{x}>0 \\ \varphi^{-}(t, \bar{x}, \bar{y}) & \text { if } \quad \bar{x}<0\end{cases}
$$

satisfying $\varphi(0, \bar{x}, \bar{y})=(\bar{x}, \bar{y})$, where $\varphi^{+}(t, \bar{x}, \bar{y})=\left(\varphi_{1}^{+}(t, \bar{x}, \bar{y}), \varphi_{2}^{+}(t, \bar{x}, \bar{y})\right)$ and $\varphi^{-}(t, \bar{x}, \bar{y})=\left(\varphi_{1}^{-}(t, \bar{x}, \bar{y}), \varphi_{2}^{-}(t, \bar{x}, \bar{y})\right)$ are the solutions of vector fields $X$ and $Y$, respectively.

Set $t^{+}(\bar{y})>0$ the smallest positive time such that $\varphi^{+}\left(t^{+}(\bar{y}), 0, \bar{y}\right) \in \Sigma$, and $t_{+}(\bar{y})<0$ be the biggest negative time such that $\varphi^{+}\left(t_{+}(\bar{y}), 0, \bar{y}\right) \in \Sigma$. Analogously, let $t^{-}(\bar{y})<0$ be the biggest negative time for which $\varphi^{-}\left(t^{-}(\bar{y}), 0, \bar{y}\right) \in \Sigma$, and $t_{-}(\bar{y})>0$ be the smallest positive time such that $\varphi^{-}\left(t_{-}(\bar{y}), 0, \bar{y}\right) \in \Sigma$. Observe that the functions $t^{+}(\bar{y}), t_{+}(\bar{y}), t^{-}(\bar{y})$, and $t_{-}(\bar{y})$ are not necessarily always defined.

Assume that $t^{+}(\bar{y})>0$ and $t^{-}(\bar{y})<0$ are defined. There exists a limit cycle passing through the point $(0, \bar{y})$ with $\bar{y} \in J^{*}=\operatorname{Dom}\left(t^{+}\right) \cap \operatorname{Dom}\left(t^{-}\right)$if and only if $\varphi_{2}^{+}\left(t^{+}(\bar{y}), 0, \bar{y}\right)=\varphi_{2}^{-}\left(t^{-}(\bar{y}), 0, \bar{y}\right)$. Thus, in this case the limit cycles are in correspondence with the zeros $y^{*}$ of the function

$$
f(\bar{y})=\varphi_{2}^{+}\left(t^{+}(\bar{y}), 0, \bar{y}\right)-\varphi_{2}^{-}\left(t^{-}(\bar{y}), 0, \bar{y}\right)
$$

on the domain $J^{*}$.

Equivalently, if $t_{+}(\bar{y})<0$ and $t_{-}(\bar{y})>0$ are defined then there exists a limit cycle passing through the point $(0, \bar{y})$ with $\bar{y} \in J_{*}=\operatorname{Dom}\left(t_{+}\right) \cap \operatorname{Dom}\left(t_{-}\right)$if 
and only if $\varphi_{2}^{+}\left(t_{+}(\bar{y}), 0, \bar{y}\right)=\varphi_{2}^{-}\left(t_{-}(\bar{y}), 0, \bar{y}\right)$. Thus, in this case we must study the zeros $y_{*}$ of the function

$$
f(\bar{y})=\varphi_{2}^{+}\left(t_{+}(\bar{y}), 0, \bar{y}\right)-\varphi_{2}^{-}\left(t_{-}(\bar{y}), 0, \bar{y}\right),
$$

on the domain $J_{*}$.

Since the vector fields $X$ and $Y$ are linear, then a limit cycle passing through the point $\left(x_{0}, y_{0}\right)$ must contain points of the form $\left(0, y^{*}\right)$ and $\left(0, y_{*}\right)$ such that $y^{*} \in J^{*}$ and $y_{*} \in J_{*}$. Therefore detecting all the zeros of (7) or (8) we must detect all the limit cycles.

In [7] the authors proved that the discontinuous piecewise linear differential systems of type $F F, F N$ and $F i N$ have at most two limit cycles. Here assuming that these systems are piecewise MY-systems we shall prove that they have at most one limit cycle.

4.1. Piecewise MY-systems of type FF. In this subsection we prove that a piecewise MY-system having at the origin a boundary stable focus of $Y$ and having $X$ a virtual stable focus have at most one limit cycle.

Assume that system (6) have a singularity of type boundary focus for $x<0$ and a virtual focus for $x>0$. By Proposition 2 the matrix corresponding to the linear vector field $X$ of (6) can be transformed into $M_{2}$ and the associated matrix of the linear vector field $Y$ is transformed is a general matrix $B=\left(b_{i j}\right)$. Then we can rewrite system (6) as

$$
\left(\begin{array}{l}
\dot{x} \\
\dot{y}
\end{array}\right)=\left\{\begin{array}{l}
\left(\begin{array}{cc}
a & 1 \\
-1 & a
\end{array}\right)\left(\begin{array}{c}
x+u_{1} \\
y+u_{2}
\end{array}\right) \quad \text { if } \quad x>0 \\
\left(\begin{array}{ll}
b_{11} & b_{12} \\
b_{21} & b_{22}
\end{array}\right)\left(\begin{array}{c}
x \\
y+v_{2}
\end{array}\right) \text { if } \quad x<0
\end{array}\right.
$$

where $4 b_{12} b_{21}+\left(b_{11}-b_{22}\right)^{2}<0$ because we have a focus for the vector field $Y$. Furthermore $u_{1}>0$ because the focus of the vector field $X$ is virtual.

Since (9) is a piecewise discontinuous linear differential system, we can compute its solution. Thus the solution $\varphi^{+}(t, \bar{x}, \bar{y})$ of $(9)$ for $\bar{x}>0$ such that $\varphi^{+}(0, \bar{x}, \bar{y})=(\bar{x}, \bar{y})$ is $\varphi^{+}(t, \bar{x}, \bar{y})=\left(\varphi_{1}^{+}(t, \bar{x}, \bar{y}), \varphi_{2}^{+}(t, \bar{x}, \bar{y})\right)$ where

$$
\begin{aligned}
& \varphi_{1}^{+}(t, \bar{x}, \bar{y})=e^{a t}\left(\left(u_{1}+\bar{x}\right) \cos t+\left(u_{2}+\bar{y}\right) \sin t\right)-u_{1}, \\
& \varphi_{2}^{+}(t, \bar{x}, \bar{y})=e^{a t}\left(\left(u_{2}+\bar{y}\right) \cos t-\left(u_{1}+\bar{x}\right) \sin t\right)-u_{2},
\end{aligned}
$$

and the solution $\varphi^{-}(t, \bar{x}, \bar{y})$ of $(9)$, for $\bar{x}<0$, such that $\varphi^{-}(0, \bar{x}, \bar{y})=(\bar{x}, \bar{y})$ is given by $\varphi^{-}(t, \bar{x}, \bar{y})=\left(\varphi_{1}^{-}(t, \bar{x}, \bar{y}), \varphi_{2}^{-}(t, \bar{x}, \bar{y})\right)$, where 


$$
\begin{aligned}
\varphi_{1}^{-}(t, \bar{x}, \bar{y})= & {\left[\sin \left(\frac{\Gamma t}{2}\right) \frac{\left(\left(b_{11}-b_{22}\right) \bar{x}+2 b_{12}\left(\bar{y}+v_{2}\right)\right)}{\Gamma}\right.} \\
& \left.+\bar{x} \cos \left(\frac{\Gamma t}{2}\right)\right] e^{\frac{\left(b_{11}+b_{22}\right) t}{2}}, \\
\varphi_{2}^{-}(t, \bar{x}, \bar{y})= & {\left[\sin \left(\frac{\Gamma t}{2}\right) \frac{\left(\left(b_{22}-b_{11}\right)\left(v_{2}+\bar{y}\right)+2 b_{21} \bar{x}\right)}{\Gamma}\right.} \\
& \left.+\left(v_{2}+\bar{y}\right) \cos \left(\frac{\Gamma t}{2}\right)\right] e^{\frac{\left(b_{11}+b_{22}\right) t}{2}}-v_{2},
\end{aligned}
$$

with $\Gamma=\sqrt{-\left(b_{11}-b_{22}\right)^{2}-4 b_{12} b_{21}}$.

We have the following result. The first part of its proof is in the proof of Proposition 11 of [7].

Theorem 4. Assume that the eigenvalues of the vector fields $X$ and $Y$ have negative real parts, then the piecewise discontinuous linear differential system (9) has at most one limit cycle.

Proof. In order to fix the clockwise orientation of the flow of system (9) we assume that $Y_{1}\left(0,1-v_{2}\right)=b_{12}>0$.

Observe that the orbits of vector field $Y$ expends a time $t^{-}(\bar{y})=-2 \pi / \Gamma$ in the half-plane $\Sigma^{-}$if $\bar{y}>-v_{2}$ or $t_{-}(\bar{y})=2 \pi / \Gamma$ if $\bar{y}<-v_{2}$, where the point $\left(0,-v_{2}\right)$ is the singularity. Therefore the functions $t^{-}(\bar{y})<0$ and $t_{-}(\bar{y})>0$ are define for $\bar{y}>-v_{2}$ and $\bar{y}<-v_{2}$, respectively.

Doing a convenient translation in the plane so that it preserves the straight line $\Sigma$ and the half-plane $x>0$, we can take $u_{2}=0$. This implies that the singularity of $X$ is $\left(-u_{1}, 0\right)$. Furthermore the point $\left(0,-a u_{1}\right) \in \Sigma$ is an invisible fold point for the vector field $X$. It follows that the function $t^{+}(\bar{y})>0$ is defined for every $\bar{y}>-a u_{1}$ (see Figure 1).

Consider $\bar{y}>-a u_{1}$ and $y=\frac{\bar{y}}{u_{1}} x+\bar{y}$ the straight line $L$ passing through the points $\left(-u_{1}, 0\right)$ and $(0, \bar{y})$. Then

$$
\left(-u_{1}\left(e^{a \pi}+1\right),-\bar{y} e^{a \pi}\right)=\varphi^{+}(\pi, 0, \bar{y}) \in L,
$$

and this implies that $\varphi^{+}(t, 0, \bar{y}) \in \Sigma$ for some $t<\pi$. Thus $t^{+}(\bar{y}) \in(0, \pi)$ for every $\bar{y}>-a u_{1}$. 


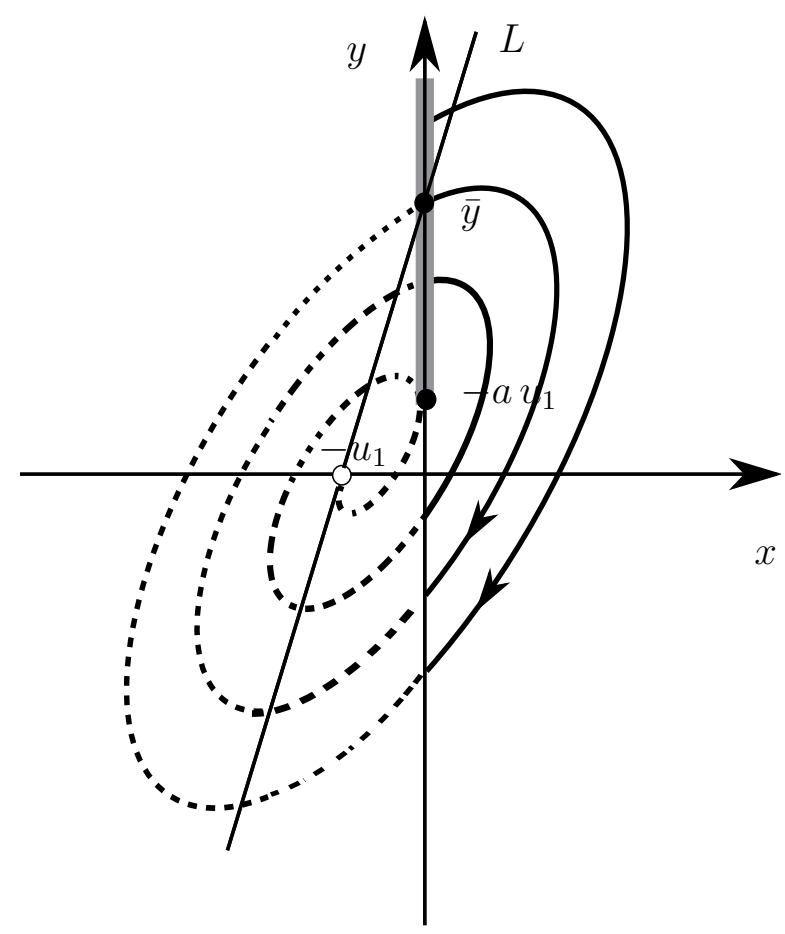

Figure 1. Virtual focus for the vector field $X$. The shaded half-line is the domain of definition of the function $t^{+}(\bar{y})>0$.

By definition of $t^{+}(\bar{y})$, we have that $\varphi_{1}^{+}\left(t^{+}(\bar{y}), 0, \bar{y}\right)=0$ for every $\bar{y}>-a u_{1}$, that is

$$
e^{a t^{+}(\bar{y})}\left(u_{1} \cos t^{+}(\bar{y})+\bar{y} \sin t^{+}(\bar{y})\right)-u_{1}=0 .
$$

Then taking $y^{+}(t)=u_{1} F(t)$, with $F$ defined in (5), for $t \in(0, \pi)$ we have that $y^{+}\left(t^{+}(\bar{y})\right)=\bar{y}$ for every $\bar{y}>-a u_{1}$. Furthermore, by Lemma 3 we have that $y^{+}(t)>-a u_{1}$ is an injective function on the interval $(0, \pi)$. It follows that given $t_{0} \in(0, \pi)$ and $y_{0}=y^{+}\left(t_{0}\right)$ we obtain $y^{+}\left(t^{+}\left(y_{0}\right)\right)=y_{0}$. Therefore $y^{+}\left(t^{+}\left(y_{0}\right)\right)=y^{+}\left(t_{0}\right)$ and this implies that $t^{+}\left(y^{+}\left(t_{0}\right)\right)=t^{+}\left(y_{0}\right)=t_{0}$. Since $t_{0}$ was arbitrarily chosen, we conclude that $t^{+}\left(y^{+}(t)\right)=t$ for every $t \in(0, \pi)$. Therefore the function $t^{+}:\left(-a u_{1}, \infty\right) \rightarrow(0, \pi)$ is invertible with inverse equal to $y^{+}:(0, \pi) \rightarrow\left(-a u_{1}, \infty\right)$.

In this case we have that the function (7) is given by

(12) $f(\bar{y})=v_{2}+e^{-\frac{\left(b_{11}+b_{22}\right) \pi}{\Gamma}}\left(v_{2}+\bar{y}\right)+e^{a t^{+}(\bar{y})}\left(\bar{y} \cos t^{+}(\bar{y})-u_{1} \sin t^{+}(\bar{y})\right)$. 
Thus, defining $y_{M}=\max \left\{-a u_{1},-v_{2}\right\}$ we obtain that the functions $t^{+}(\bar{y})$ and $t^{-}(\bar{y})$ are defined for every $\bar{y}>y_{M}$. We conclude that computing the zeros of the function (12) for $\bar{y}>y_{M}$ is equivalent to compute the zeros of the function $g_{1}(t)=f\left(y^{+}(t)\right)$ given by

$$
g_{1}(t)=v_{2}(1+\delta)+u_{1}\left(\cot t-e^{a t} \csc t\right)-\delta u_{1}\left(\cot t-e^{-a t} \csc t\right),
$$

for $t \in I=t^{+}\left(\left(Y_{M}, \infty\right)\right) \subset(0, \pi)$ and $\delta=e^{-\left(b_{11}+b_{22}\right) \pi / \Gamma}$. Since the eigenvalues of $Y$ are negative we have that $\delta>1$. Moreover $I=(0, \pi)$ provided $v_{2} \geq a u_{1}$.

We will prove that the function (13) has at most one zero for $t \in(0, \pi)$.

Notice that, for $t \in(0, \pi), g_{1}(t)=0$ is equivalent to

$$
A \sin t+(1-\delta) \cos t-e^{a t}+\delta e^{-a t}=0
$$

where $A=\frac{v_{2}(1+\delta)}{u_{1}}$. Defining $h(t)=(1-\delta) \cos t-e^{a t}+\delta e^{-a t}$, we get

$$
h^{\prime}(t)=-(1-\delta) \sin t-a e^{a t}-a \delta e^{-a t}>0,
$$

for $t \in(0, \pi)$, because $\delta>1$ and $a<0$. This implies that $h(t)$ is increasing in $(0, \pi)$. Since $h(0)=0$, we have that $h(t)>0$ in $(0, \pi)$. Therefore if $A>0$ equation (14) does not have solution in $(0, \pi)$ and, consequently the function (13) does not have zeros.

In what follows we assume $A<0$. Consider the functions

$$
\begin{aligned}
& h_{1}(t)=-A \sin t-(1-\delta) \cos t, \\
& h_{2}(t)=-e^{a t}+\delta e^{-a t},
\end{aligned}
$$

and note that equation (14) holds if and only if $h_{1}(t)=h_{2}(t)$. We will prove that there exists at most one $\bar{t} \in(0, \pi)$ such that $h_{1}(\bar{t})=h_{2}(\bar{t})$.

Observe that $h_{1}(0)=\delta-1, h_{1}(\pi / 2)=-A, h_{1}(\pi)=1-\delta$, and $h_{1}\left(t_{0}\right)=0$, where $t_{0}=\arctan \left(\frac{\delta-1}{A}\right)$. Furthermore,

$$
\begin{aligned}
& h_{1}^{\prime}(t)=-A \cos t+(1-\delta) \sin t, \\
& h_{1}^{\prime \prime}(t)=A \sin t+(1-\delta) \cos t,
\end{aligned}
$$

where $h_{1}^{\prime}(t)>0$ (respectively $<$ ) for every $t<t_{1}=\arctan (A /(1-\delta))$ (respectively $>$ ) and $h_{1}^{\prime \prime}(t)<0$ ( respectively $>$ ) for every $t<t_{0}$ (respectively $>$ ). Notice that $t_{0} \in\left(\frac{\pi}{2}, \pi\right)$ and $t_{1} \in\left(0, \frac{\pi}{2}\right)$. It follows that the graph of $h_{1}(t)$ is given in Figure 2-(a). 

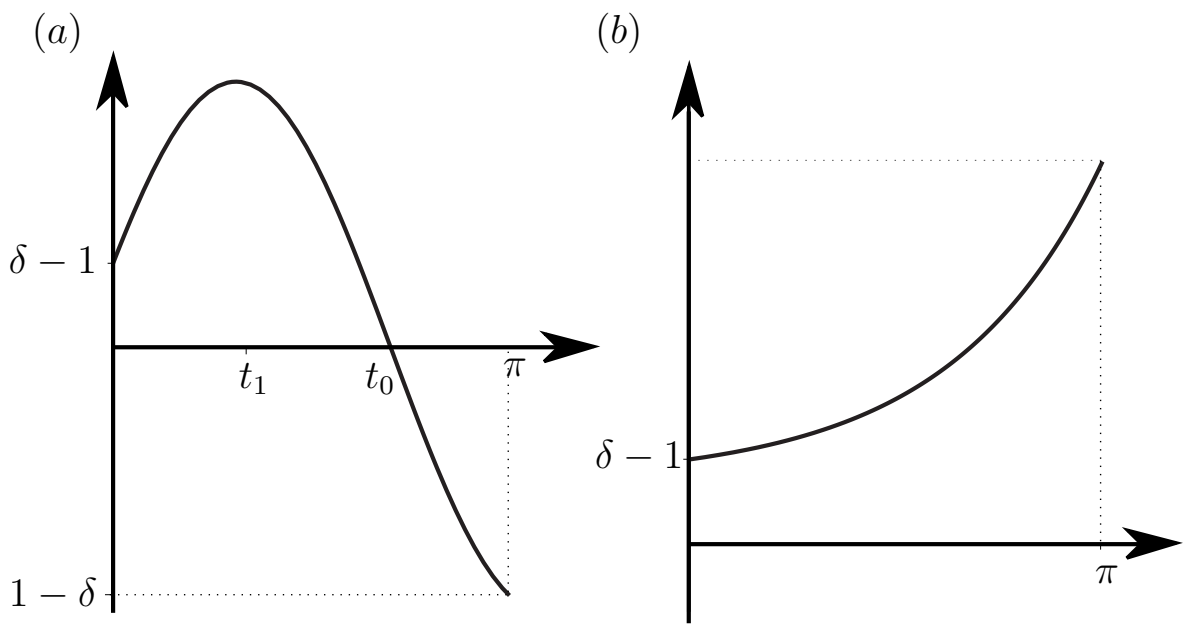

Figure 2. (a) Graph of the function $h_{1}(t) ;(b)$ Graph of the function $h_{2}(t)$.

Since $h_{2}^{\prime}(t)>0$ for $t \in(0, \pi)$ we have $h_{2}(t)$ is increasing in $t \in(0, \pi)$, and $h_{2}(0)=\delta-1>0$. Furthermore,

$$
h_{2}^{\prime \prime}(t)=-a^{2} e^{a t}+a^{2} \delta e^{-a t}=a^{2} e^{a t}\left(-1+\delta e^{-2 a t}\right)>0
$$

for $t \in(0, \pi)$. The graph of the function $h_{2}(t)$ is given in Figure 2-(b).

Analyzing the graphs of the functions $h_{1}(t)$ and $h_{2}(t)$ and taking into account their convexities, we conclude that if $h_{2}^{\prime}(0)<h_{1}^{\prime}(0)$, then there exists a unique $\bar{t} \in\left(0, t_{0}\right) \subset(0, \pi)$ such that $h_{1}(\bar{t})=h_{2}(\bar{t})$. If $h_{2}^{\prime}(0) \geq h_{1}^{\prime}(0)$ then $h_{2}(t)>h_{1}(t)$ for every $t \in(0, \pi)$. Therefore equation (14) has at most one zero in $(0, \pi)$.

Corollary 5. A piecewise $M Y$-system of type FF has at most one limit cycle.

Proof. The result follows applying Theorem 4 to piecewise MY-systems whose singularities for the vector fields $X$ and $Y$ are a virtual stable focus and a boundary stable focus at the origin, respectively.

The following is an example of a piecewise MY-system of type $F F$ having one limit cycle. With this example Theorem 1 is proved for a piecewise MYsystems of type $F F$. 
Example 6. Consider the following piecewise discontinuous linear differential system

$$
\dot{z}= \begin{cases}X(z)=\left(y-\frac{x}{2}-\frac{1}{10},-x-\frac{y}{2}-\frac{1}{5}\right) \quad \text { for } x>0, \\ Y(z)=\left(y-x-1,-\frac{\pi^{2} x}{(\ln 2)^{2}}-y+1\right) & \text { for } x<0 .\end{cases}
$$

Observe that the point $(-1 / 5,0)$ is a virtual focus of $X$, and that the point $(0,1)$ is a boundary focus of $Y$. Furthermore the eigenvalues of (16) are $\lambda_{1,2}^{+}=-\frac{1}{2} \pm i$ for the point $(-1 / 5,0)$, and $\lambda_{1,2}^{-}=-1 \pm \frac{\pi}{\ln 2} i$, for the point $(0,1)$. Thus, by Theorem 4 system (16) has at most one limit cycle.

In this case we have that $y_{M}=\max \{1 / 10,1\}=1, y^{+}(t)=\left(e^{t / 2} \csc t-\right.$ $\cot t) / 5$, and the function defined in (13) is

$$
g_{1}(t)=-3-\frac{1}{5} \cot t+\frac{2 e^{t / 2}}{5} \csc t-\frac{e^{-t / 2}}{5} \csc t
$$

Thus in the interval $(0, \pi)$ we have that $g_{1}(t)=0$ if and only if $t \approx 2.588 \ldots$ and, therefore system $(16)$ has one limit cycle passing thorough $y^{+}(2.588 \ldots) \approx$ $1.714 \ldots>y_{M}$.

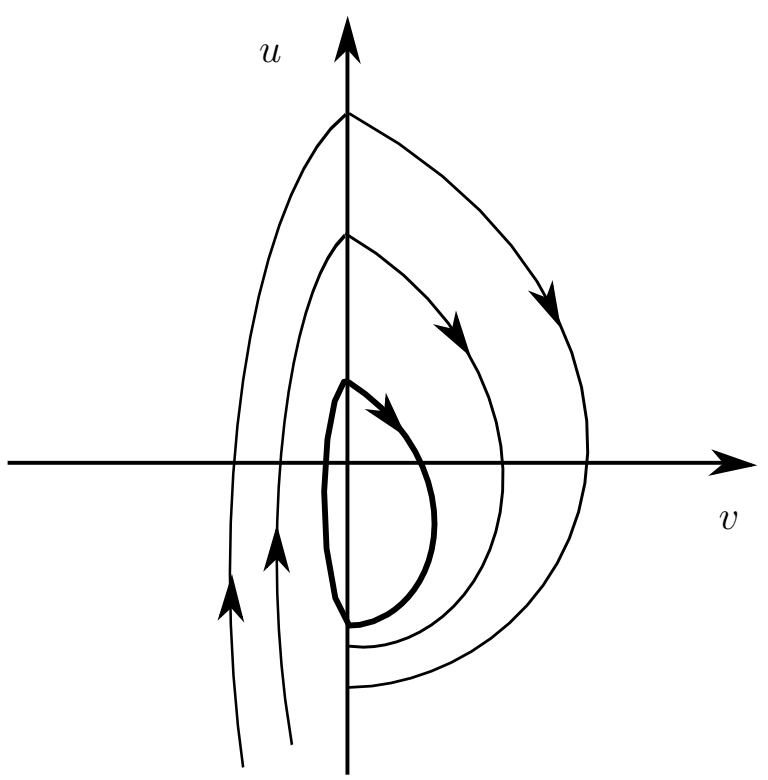

FiguRE 3. The phase portrait of the piecewise MY-system (17). 
Changing the coordinates to $u=x$ and $v=y-1$, we rewrite the system (16) as

$$
\dot{z}= \begin{cases}\left(v-\frac{u}{2}+\frac{9}{10},-u-\frac{v}{2}-\frac{7}{10}\right) & \text { for } u>0 \\ \left(v-u,-\frac{\pi^{2} u}{(\ln 2)^{2}}-v\right) & \text { for } u<0\end{cases}
$$

whose boundary focus is at $(0,0)$ and the virtual focus is at $(-1 / 5,-1)$. Observe that system (17) is a piecewise $M Y$-system and it has one limit cycle passing through point $(u, v)=(0,0.714 \ldots)$, see Figure 3.

4.2. Piecewise MY-systems of type $F N$. In this subsection we prove that a piecewise MY-system having at the origin a boundary stable focus of $Y$ and having $X$ a virtual stable node $N$ have at most one limit cycle.

Suppose that the piecewise discontinuous linear differential system (6) has a virtual node $N$ for $x>0$ and a boundary focus for $x<0$. By Proposition 2 the matrix of the linear vector field $X$ can be transformed into $M_{1}$ and the matrix of the vector field $Y$ is a general matrix $B=\left(b_{i j}\right)$. Therefore system (6) after this transformation becomes

$$
\left(\begin{array}{l}
\dot{x} \\
\dot{y}
\end{array}\right)=\left\{\begin{array}{l}
\left(\begin{array}{ll}
a & 1 \\
1 & a
\end{array}\right)\left(\begin{array}{c}
x+u_{1} \\
y+u_{2}
\end{array}\right) \quad \text { if } \quad x>0, \\
\left(\begin{array}{ll}
b_{11} & b_{12} \\
b_{21} & b_{22}
\end{array}\right)\left(\begin{array}{c}
x \\
y+v_{2}
\end{array}\right) \text { if } \quad x<0
\end{array}\right.
$$

where $4 b_{12} b_{21}+\left(b_{11}-b_{22}\right)^{2}<0$. Note that $u_{1}>0$ because the vector field $X$ has a virtual node.

The solution $\varphi^{+}(t, \bar{x}, \bar{y})$ of $(18)$ with $\bar{x}>0$, such that $\varphi^{+}(0, \bar{x}, \bar{y})=(\bar{x}, \bar{y})$ is $\varphi^{+}(t, \bar{x}, \bar{y})=\left(\varphi_{1}^{+}(t, \bar{x}, \bar{y}), \varphi_{2}^{+}(t, \bar{x}, \bar{y})\right)$ where

$$
\begin{aligned}
& \varphi_{1}^{+}(t, \bar{x}, \bar{y})=-u_{1}+e^{a t}\left[\left(u_{1}+\bar{x}\right) \cosh t+\left(u_{2}+\bar{y}\right) \sinh t\right] \\
& \varphi_{2}^{+}(t, \bar{x}, \bar{y})=-u_{2}+e^{a t}\left[\left(u_{2}+\bar{y}\right) \cosh t+\left(u_{1}+\bar{x}\right) \sinh t\right] .
\end{aligned}
$$

The solution of $(18)$ with $\bar{x}<0$ is $\varphi^{-}(t, \bar{x}, \bar{y})=\left(\varphi_{1}^{-}(t, \bar{x}, \bar{y}), \varphi_{2}^{-}(t, \bar{x}, \bar{y})\right)$, where $\varphi_{1}^{-}$and $\varphi_{2}^{-}$are given in (10) and (11), respectively.

Assuming that the real parts of the eigenvalues of system (18) are negative, that is $a<-1$ and $b_{11}+b_{22}<0$, we will prove that system (18) has at most 
one limit cycle. In order to fix the clockwise orientation of the orbits of system (18) we assume that $Y_{1}\left(0,1-v_{2}\right)=b_{12}>0$.

The first part of the proof of the next result is in the proof of Proposition 14 of [7].

Theorem 7. Assume that the eigenvalues of vector fields $X$ and $Y$ have negative real parts, then the piecewise discontinuous linear differential system (18) has at most one limit cycle.

Proof. Doing a translation to system (18) that preserves the half-plane $x>0$ and the discontinuity line $\Sigma$, we can assume that $u_{2}=0$.

Clearly the point $\left(0,-a u_{1}\right) \in \Sigma$ is an invisible fold point, and $\left(-u_{1}, 0\right)$ is the singularity of $X$. Furthermore the invariant straight lines of the node intersect the line $\Sigma$ at the points $\left(0, y^{s}\right)$ and $\left(0, y^{s s}\right)$, respectively, where $y^{s}=u_{1}<-a u_{1}$ and $y^{s s}=-u_{1}<u_{1}$. It follows that the function $t^{+}(y)>0$ is defined for every $y>-a u_{1}$, see Figure 4 .

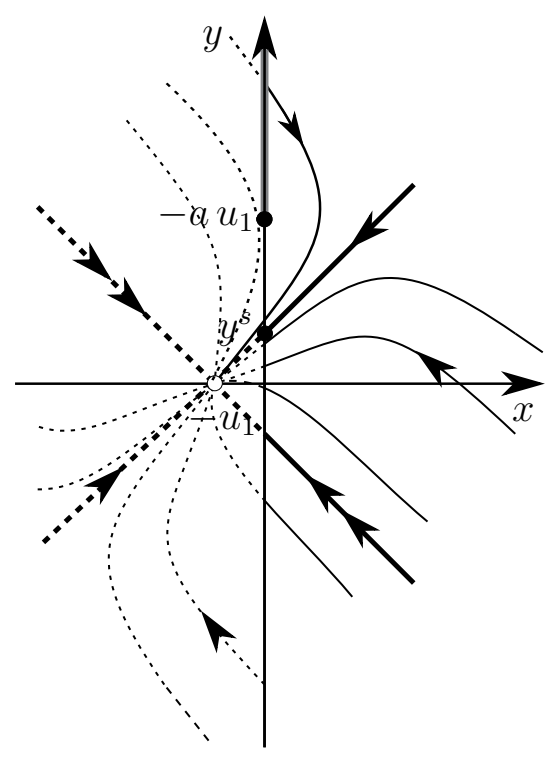

Figure 4. Virtual diagonalizable node of the vector field $X$.

The shaded line is the domain of definition of the function $t^{+}(\bar{y})>0$.

By definition of $t^{+}(\bar{y})$, from (19) we get that

$$
\varphi_{1}^{+}\left(t^{+}(\bar{y}), 0, \bar{y}\right)=-u_{1}+e^{a t^{+}(\bar{y})}\left(u_{1} \cosh t^{+}(\bar{y})+\bar{y} \sinh t^{+}(\bar{y})\right)=0,
$$

for every $\bar{y}>-a u_{1}$. Thus defining $y^{+}(t)=u_{1} G(t)$ for $t>0$, with $G(t)$ given in (5), we have that $y^{+}\left(t^{+}(\bar{y})\right)=\bar{y}$ for every $\bar{y}>-a u_{1}$. 
Computing implicitly the derivative in the variable $\bar{y}$ of the identity $y^{+}\left(t^{+}(\bar{y})\right)=$ $\bar{y}$ we obtain

$$
\begin{aligned}
\frac{d t^{+}(\bar{y})}{d \bar{y}} & =\frac{\sinh t^{+}(\bar{y})}{u_{1} \operatorname{csch} t^{+}(\bar{y})-u_{1} e^{-a t^{+}(\bar{y})}\left(a+\operatorname{coth} t^{+}(\bar{y})\right)} \\
& =\frac{\sinh ^{2} t^{+}(\bar{y})}{u_{1}\left[1-\left(a \sinh t^{+}(\bar{y})+\cosh t^{+}(\bar{y})\right) e^{-a t^{+}(\bar{y})}\right]}>0
\end{aligned}
$$

for $t^{+}(\bar{y})>0$, because $a<-1$ and $u_{1}>0$.

We claim that $t^{+}\left(y^{+}(t)\right)=t$ for every $t>0$. Consider $y_{0}=y^{+}\left(t_{0}\right)$ for some $t_{0}>0$. From Lemma 3 we have that the function $y^{+}(t)$ is injective on $\mathbb{R}^{+}$and $y^{+}(t)>-a u_{1}$. Therefore $y_{0}>-a u_{1}$ and from the previous results $y_{0}=y^{+}\left(t^{+}\left(y_{0}\right)\right)$. Thus $y^{+}\left(t_{0}\right)=y^{+}\left(t^{+}\left(y_{0}\right)\right)$ from which it follows that $t_{0}=$ $t^{+}\left(y_{0}\right)=t^{+}\left(y^{+}\left(t_{0}\right)\right)$. Since $t_{0}>0$ was arbitrarily chosen we conclude that $t^{+}\left(y^{+}(t)\right)=t$ for every $t>0$. Therefore the function $t^{+}:\left(-a u_{1}, \infty\right) \rightarrow \mathbb{R}^{+}$is invertible with inverse equal to $y^{+}: \mathbb{R}^{+} \rightarrow\left(-a u_{1}, \infty\right)$.

Computing the zeros of the function (7) for $y>Y_{M}=\max \left\{-a u_{1},-v_{2}\right\}$ is equivalent to compute the zeros of the function

$$
g_{2}(t)=f\left(y^{+}(t)\right)=v_{2}(1+\delta)+u_{1}(1-\delta) \operatorname{coth} t-u_{1}\left(e^{a t}-\delta e^{-a t}\right) \operatorname{csch} t
$$

for $t \in I=t^{+}\left(\left(Y_{M}, \infty\right)\right) \subset \mathbb{R}^{+}$and $\delta=e^{-\left(b_{11}+b_{22}\right) \pi / \Gamma}$. Moreover $I=\mathbb{R}^{+}$ provided $v_{2} \geq a u_{1}$.

We will prove that the function $g_{2}(t)$ has at most one zero for $t>0$.

Notice that $\delta>1$ and $g_{2}(t)=0$ is equivalent to

$$
A \sinh t+(1-\delta) \cosh t-e^{a t}+\delta e^{-a t}=0,
$$

where $A=\frac{v_{2}(1+\delta)}{u_{1}}$.

Consider the functions

$$
\begin{aligned}
& h_{2}(t)=-e^{a t}+\delta e^{-a t}, \\
& h_{3}(t)=-A \sinh t-(1-\delta) \cosh t,
\end{aligned}
$$

with $t>0$, and note that equation (21) holds if and only if $h_{2}(t)=h_{3}(t)$. We will prove that there exists at most one $\bar{t} \in \mathbb{R}^{+}$such that $h_{2}(\bar{t})=h_{3}(\bar{t})$.

By the proof of Theorem 4 we have that graph of $h_{2}(t)$ is given in Figure 2. Observe that

$$
\begin{aligned}
& h_{3}^{\prime}(t)=-A \cosh t+(\delta-1) \sinh t, \\
& h_{3}^{\prime \prime}(t)=-A \sinh t+(\delta-1) \cosh t,
\end{aligned}
$$


from which it follows that, if $A<0$ then $h_{3}^{\prime}(t)>0$ and $h_{3}^{\prime \prime}(t)>0$. Furthermore we have that $h_{3}(0)=\delta-1>0$, so $h_{3}(t)$ is a positive strictly increasing function and its graph is given in Figure 5-(a). On the other hand if $A>0$ we have three cases to consider

(1) $A<\delta-1$. In this case $h_{3}(t) \neq 0$ and $h_{3}^{\prime}\left(t_{1}\right)=0$, where $t_{1}=$ $\frac{1}{2} \ln \left(-\frac{A+\delta-1}{A+1-\delta}\right)$, and $h_{3}^{\prime \prime}(t)>0$. Consequently the graph of the function $h_{3}(t)$ is given in Figure $5-(b)$.

(2) $A>\delta-1$. In this case we obtain that $h_{3}\left(t_{0}\right)=0$, where $t_{0}=$ $\frac{1}{2} \ln \left(\frac{A+\delta-1}{A+1-\delta}\right), h_{3}^{\prime}(t)<0$ and $h_{3}^{\prime \prime}\left(t_{0}\right)>0$ (respectively $<$ ) for every $t<t_{0}$ (respectively $>$ ). It follows that the graph of the function $h_{3}(t)$ is given in Figure $5-(c)$.

(3) $A=\delta-1$. We have that $h_{3}(t)>0, h_{3}^{\prime}(t)<0$ and $h_{3}^{\prime \prime}(t)>0$, and this implies that the function $h_{3}(t)$ is strictly decreasing for $t>0$. The graph of the function $h_{3}(t)$ is given in Figure $5-(d)$.

(a)

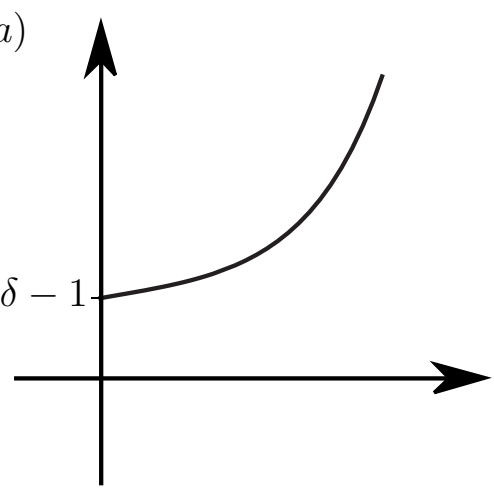

(c)

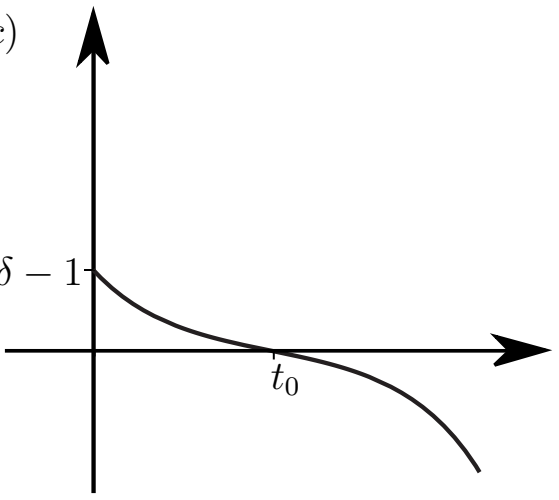

(b)

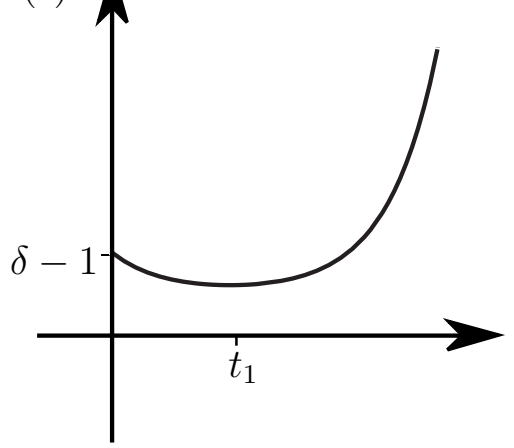

(d)

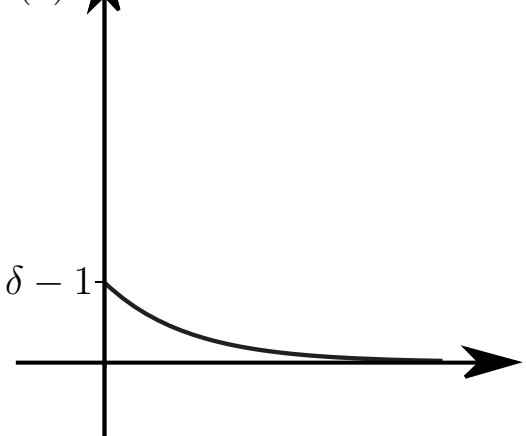

Figure 5. Graph of $h_{3}(t):$ (a) $A<0$, (b) $0<A<\delta-1$, (c) $A>\delta-1$, and $(d) A=\delta-1$. 
Analyzing the graph of the functions $h_{2}(t)$ and $h_{3}(t)$ we obtain that, for $A<\delta-1$ there exists at most one $\bar{t} \in \mathbb{R}^{+}$such that $h_{2}(\bar{t})=h_{3}(\bar{t})$, and for $A \geq \delta-1$ we have $h_{2}(t) \neq h_{3}(t)$ for every $t \in \mathbb{R}^{+}$. Therefore, equation (21) holds for at most one $t \in \mathbb{R}^{+}$and, consequently the function $g_{2}(t)$ have at most one zero $t \in \mathbb{R}^{+}$.

Corollary 8. A piecewise $M Y$-system of type $F N$ have at most one limit cycle.

Proof. The result follows applying Theorem 7 to piecewise MY-systems whose singularities for the vector fields $X$ and $Y$ are a virtual diagonalizable stable node and a boundary stable focus at the origin, respectively.

The following is an example of piecewise a MY-system of type $F N$ having one limit cycle.

\section{Example 9. Let}

$$
\dot{z}= \begin{cases}X(z)=\left(y-2 x-\frac{2}{5}, x-2 y+\frac{1}{5}\right) & \text { for } x>0, \\ Y(z)=\left(y-\frac{x}{\pi} \ln \left(\frac{3}{2}\right)-1,-x-\ln \left(\frac{3}{2}\right)\left(\frac{y}{\pi}-\frac{1}{\pi}\right)\right) & \text { for } x<0,\end{cases}
$$

be a piecewise discontinuous linear differential system. The singularities of (22) are $(-1 / 5,0)$ which is a virtual node of $X$, and $(0,1)$ which is a boundary focus of $Y$. Observe that, the eigenvalues of (22) are $\lambda_{1}^{+}=-1$ and $\lambda_{2}^{+}=-3$, for the point $(-1 / 5,0)$, and $\lambda_{1,2}^{-}=\frac{1}{\pi} \ln \left(\frac{3}{2}\right) \pm i$, for the point $(0,1)$. Thus, by Theorem 7 system (22) has at most one limit cycle.

In this case we have that $y_{M}=\max \{1,2 / 5\}=1, y^{+}(t)=\frac{e^{2 t}}{5} \operatorname{csch} t-\frac{\operatorname{coth} t}{5}$, and the function defined in (20) is

$$
g_{2}(t)=-\frac{5}{2}-\frac{\operatorname{coth} t}{10}-\frac{e^{-2 t}}{5} \operatorname{csch} t+\frac{3 e^{2 t}}{10} \operatorname{csch} t .
$$

Then for $t>0, g_{2}(t)=0$ if and only if $t \approx 1.412 \ldots$ and, therefore system $(22)$ has one limit cycle passing thorough $y^{+}(1.412 \ldots) \approx 1.520 \ldots>y_{M}$.

Changing the coordinates to $u=x$ and $v=y-1$, we rewrite system (22) as

$$
\dot{z}= \begin{cases}\left(v-2 u+\frac{3}{5}, u-2 v-\frac{9}{5}\right) & \text { for } \quad u>0 \\ \left(v-\frac{u}{\pi} \ln \frac{3}{2},-u-\frac{v}{\pi} \ln \frac{3}{2}\right) & \text { for } \quad u<0\end{cases}
$$




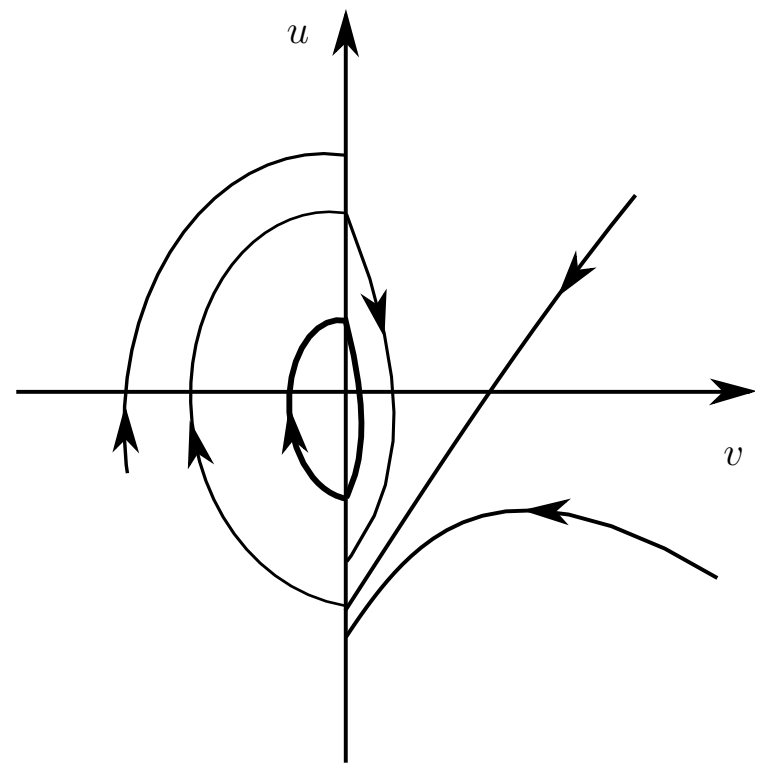

Figure 6 . The phase portrait of the piecewise MY-system (23).

whose boundary focus is at $(0,0)$ and the virtual focus is at $(-1 / 5,-1)$. We conclude that system (23) is a piecewise $M Y$-system and it has one limit cycle passing through point $(u, v)=(0,0.520 \ldots)$, see Figure 6 .

4.3. The piecewise MY-system of type FiN. In this subsection we prove that the piecewise MY-systems $Z=(X, Y)$ having at the origin a boundary stable focus of $Y$ and having $X$ a stable virtual node $i N$ has at most one limit cycle.

Suppose that $X$ has a virtual improper node $i N$, and $Y$ has a boundary focus. By Proposition 2 the matrix of the linear vector field $X$ is transformed into $M_{3}$ and the matrix of the linear vector field $Y$ is a general matrix $B=\left(b_{i j}\right)$. Therefore we rewrite system (6) as

$$
\left(\begin{array}{l}
\dot{x} \\
\dot{y}
\end{array}\right)=\left\{\begin{array}{l}
\left(\begin{array}{cc}
\lambda & \lambda \\
0 & \lambda
\end{array}\right)\left(\begin{array}{c}
x+u_{1} \\
y+u_{2}
\end{array}\right) \\
\left(\begin{array}{ll}
b_{11} & b_{12} \\
b_{21} & b_{22}
\end{array}\right)\left(\begin{array}{c}
x \\
y+v_{2}
\end{array}\right) \text { if } \quad x>0,
\end{array}\right.
$$

where $\lambda= \pm 1$ and $4 b_{12} b_{21}+\left(b_{11}-b_{22}\right)^{2}<0$. Furthermore $u_{1}>0$ because the vector field $X$ has a virtual improper node for system (24). 
In order to fix the clockwise orientation of the orbits of vector field $Y$ we assume that $Y_{1}\left(0,1-v_{2}\right)=b_{12}>0$.

The solution of $(24)$ for $\bar{x}<0$ is $\varphi^{-}(t, \bar{x}, \bar{y})=\left(\varphi_{1}^{-}(t, \bar{x}, \bar{y}), \varphi_{2}^{-}(t, \bar{x}, \bar{y})\right)$ where $\varphi_{1}^{-}$and $\varphi_{2}^{-}$are given by equations (10) and (11), respectively.

Applying a translation that preserves the line $\Sigma$ and the half-plane $\bar{x}>0$, we can assume that $u_{2}=0$. The solution $\varphi^{+}(t, \bar{x}, \bar{y})$ of $(24)$ for $\bar{x}<0$, such that $\varphi^{+}(0, \bar{x}, \bar{y})=(\bar{x}, \bar{y})$ is given by $\varphi^{+}(t, \bar{x}, \bar{y})=\left(\varphi_{1}^{+}(t, \bar{x}, \bar{y}), \varphi_{2}^{+}(t, \bar{x}, \bar{y})\right)$ where

$$
\begin{aligned}
& \varphi_{1}^{+}(t, \bar{x}, \bar{y})=-u_{1}+e^{\lambda t}\left(\lambda t \bar{y}+u_{1}+\bar{x}\right), \\
& \varphi_{2}^{+}(t, \bar{x}, \bar{y})=\bar{y} e^{\lambda t} .
\end{aligned}
$$

We have the following result.

Theorem 10. Assume that the vector fields $X$ and $Y$ have only eigenvalues with negative real part, then the piecewise discontinuous differential system (24) have at most one limit cycle.

Proof. Observe that, if $\lambda=-1$ the first return map would not be defined, because we fixed the clockwise orientation of the orbits of vector field $Y$, and there would not exist limit cycles. Therefore we assume that $\lambda=1$, and this implies that the linear vector field $X$ has an unstable singularity. Thus, we assume that the singularity of $Y$ also is unstable. We shall prove that the system (24) has at most one cycle limit and our result will follow by rescaling time $\tau=-t$.

In what follows we assume that the real parts of the eigenvalues of system (24) are positive and we prove that system (24) has at most one limit cycle.

Observe that $\left(-u_{1}, 0\right)$ is the improper node and $\left(0,-u_{1}\right)$ is an invisible fold point of $X$. The invariant straight line of the node intersects $\Sigma$ at the origin, see Figure 7. Thus the function $t_{+}(y)<0$ is defined for every $y<-u_{1}$.

Defining $y_{+}(t)=u_{1} H(t)$ for $t<0$, we have that $y_{+}(t)<-u_{1}$, because by Lemma 3 we have $H(t)<-1$. Furthermore since

$$
\varphi_{1}^{+}\left(t_{+}(\bar{y}), 0, \bar{y}\right)=-u_{1}+e^{t_{+}(\bar{y})}\left(u_{1}+\bar{y} t_{+}(\bar{y})\right)=0,
$$

for every $\bar{y}<-u_{1}$, we have that

$$
y_{+}\left(t_{+}(\bar{y})\right)=\frac{u_{1}\left(e^{-t_{+}(\bar{y})}-1\right)}{t_{+}(\bar{y})}=\bar{y}
$$

for every $\bar{y}<-u_{1}$. 


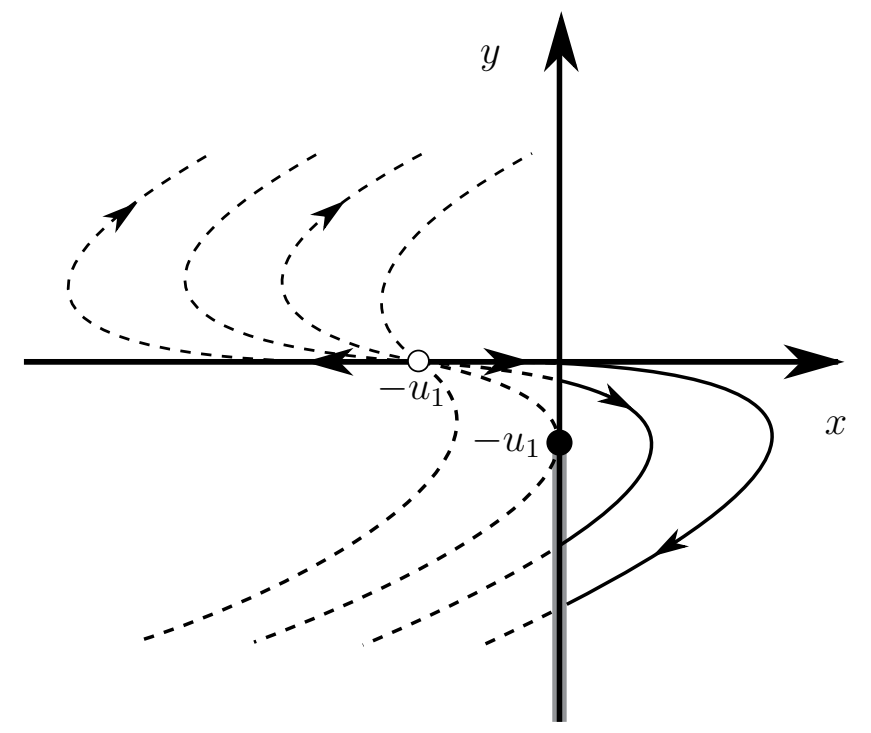

FiguRE 7. Virtual node $i N$ for the vector field $X$ when $\lambda=1$. In this case the shaded line represents the domain of the function $t_{+}(\bar{y})<0$.

We claim that the image of the function $t_{+}(\bar{y})$ is $\mathbb{R}^{-}=(-\infty, 0)$ and $t_{+}\left(y_{+}(t)\right)=$ $t$. Indeed, computing the derivative of $t_{+}(\bar{y})$ we obtain

$$
\frac{d t_{+}(\bar{y})}{d \bar{y}}=\frac{e^{t_{+}(\bar{y})} t_{+}(\bar{y})^{2}}{u_{1}\left(e^{t_{+}(\bar{y})}-t_{+}(\bar{y})-1\right)}>0,
$$

for $t_{+}(\bar{y})<0$. So $t_{+}(\bar{y})$ is strictly increasing with $t_{+}\left(-u_{1}\right)=0$ and this implies that $t_{+}(\bar{y})<0$ for every $\bar{y}<-u_{1}$. Furthermore, by previous comments, taking $t_{0}<0$ and $y_{0}=y_{+}\left(t_{0}\right)$, we have that $y_{0}<0$ and $y_{0}=y_{+}\left(t_{+}\left(y_{0}\right)\right)$. By Lemma 3 we have that $y_{+}(t)$ is injective and this implies that $t_{0}=t_{+}\left(y_{0}\right)$ and, therefore $t_{+}\left(y_{+}(t)\right)=t$ because we chose $t_{0}$ arbitrarily. Consequently $t_{+}:\left(-\infty,-u_{1}\right) \longrightarrow \mathbb{R}^{-}$is an invertible function whose inverse function is $y_{+}: \mathbb{R}^{-} \longrightarrow\left(-\infty,-u_{1}\right)$.

Define $y_{m}=\min \left\{-u_{1},-v_{2}\right\}$, then computing the zeros of the function (8) for $y<y_{m}$ is equivalent to compute the zeros of the

$$
g_{3}(t)=v_{2}(1+\delta)+\frac{u_{1}\left(1-e^{t}\right)}{t}-\frac{\delta u_{1}\left(1-e^{-t}\right)}{t},
$$

for $t \in I=y_{+}\left(-\infty, y_{m}\right) \subset \mathbb{R}^{-}$, where $\delta=e^{\left(b_{11}+b_{22}\right) \pi / \Gamma}$. Note that if $v_{2} \geq u_{1}$ then $I=\mathbb{R}^{-}$.

In what follows we prove that the function (25) has at most one zero in $\mathbb{R}^{-}$. 
Since the singularity of $Y$ is unstable we have that $\delta>1$. Moreover $g_{3}(t)=0$ is equivalent to

$$
A t+1-\delta-e^{t}+\delta e^{-t}=0
$$

where $A=\frac{v_{2}(1+\delta)}{u_{1}}$. We will prove that there exists at most one $t \in \mathbb{R}^{-}$such that equation $(26)$ holds.

(a)

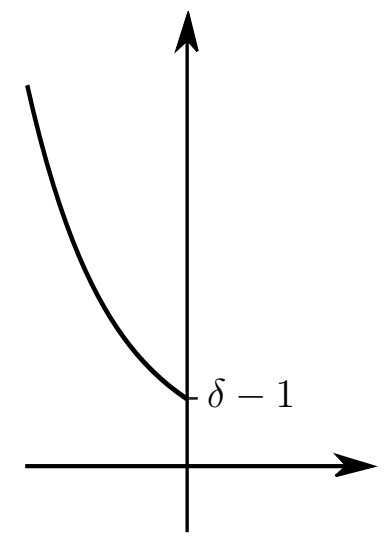

$(b)$

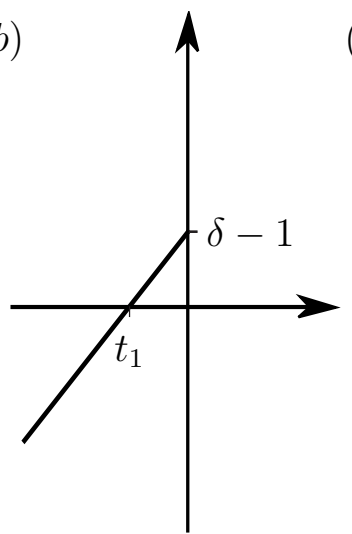

(c)

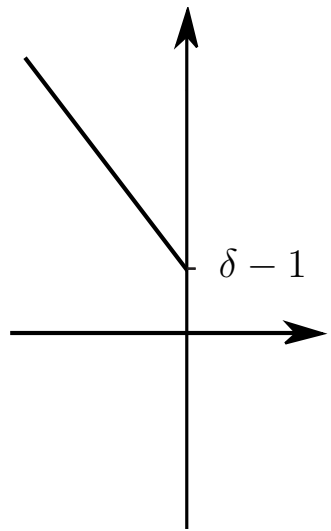

Figure 8. (a) Graph of the function $h_{2}(t),(b)$ Graph of the function $h_{1}(t)$ for $A<0$, and $(c)$ Graph of the function $h_{1}(t)$ for $A>0$.

Consider the following functions

$$
\begin{aligned}
& h_{1}(t)=-A t+\delta-1, \\
& h_{2}(t)=-e^{t}+\delta e^{-t},
\end{aligned}
$$

defined for every $t \in \mathbb{R}^{-}$and note that equation (26) holds if and only if $h_{1}(t)=h_{2}(t)$. We will show that there exists at most one $\bar{t} \in \mathbb{R}^{-}$such that $h_{1}(\bar{t})=h_{2}(\bar{t})$.

Observe that $h_{2}(t)>0$ for every $t<0, h_{2}(0)=\delta-1>0$ and $h_{2}^{\prime}(t)=$ $-e^{t}-\delta e^{-t}<0$. Then $h_{2}(t)$ is strictly decreasing positive function in $\mathbb{R}^{-}$. Furthermore $h_{2}^{\prime \prime}(t)=-e^{t}+\delta e^{-t}>0$ implies that $h_{2}(t)$ does not have inflexion points and its graph is given in Figure 8-(a).

Computing the derivative of the function $h_{1}(t)$ we obtain $h_{1}^{\prime}(t)=-A$. Furthermore we have that $h_{1}(0)=\delta-1>0$ and $h_{1}\left(t_{1}\right)=0$, where $t_{1}=(\delta-1) / A$. Therefore if $A<0$ then $h_{1}(t)$ is a strictly increasing function in $\mathbb{R}^{-}$and the graph of $h_{1}$ is given in Figure 8-(b). So there is not solution for equation $h_{1}(t)=h_{2}(t)$. On the other hand, if $A>0$ then $t_{1}>0$ and $h_{1}(t)$ is a strictly decreasing function and its graph is given by Figure $8-(c)$. 
Now analyzing the graphs of the functions $h_{1}(t)$ and $h_{2}(t)$ we conclude that to $A>0$ if $h_{1}^{\prime}(0)<h_{2}^{\prime}(0)$ there exists a unique $\bar{t} \in \mathbb{R}^{-}$such that $h_{1}(\bar{t})=h_{2}(\bar{t})$, and if $h_{1}^{\prime}(0)>h_{2}^{\prime}(0)$ then $h_{1}(t)<h_{2}(t)$. Hence there exists at most one zero of the function (25) in $\mathbb{R}^{-}$. Therefore system (24) has at most one limit cycle

Corollary 11. The piecewise $M Y$-system of type FiN has at most one limit cycle.

Proof. The result follows applying Theorem 10 to piecewise MY-systems whose singularities for the vector fields $X$ and $Y$ are a virtual improper stable node and a boundary stable focus at the origin, respectively.

In what follows we present an example of a piecewise MY-system of type FiN having one limit cycle.

Example 12. Consider the piecewise discontinuous linear differential system

$$
\dot{z}= \begin{cases}X(z)=\left(x+y+\frac{1}{2}, y\right) & \text { for } x>0, \\ Y(z)=\left(x+y+5, y-\frac{\pi^{2}}{\ln (2)^{2}} x+5\right) & \text { for } x<0 .\end{cases}
$$

The singularities of $(28)$ are $(-1 / 2,0)$, which is a virtual improper node of $X$, and $(0,-5)$, which is a boundary focus of $Y$. Observe that the eigenvalues of (28) are $\lambda^{+}=1$ for $x>0$, and $\lambda_{1,2}^{-}=1 \pm \frac{\pi}{\ln 2} i$ for $x<0$.

In this case we have that $y_{m}=\min \{-1 / 2,-5\}=-5$,

$$
y^{+}(t)=\frac{e^{-t}-1}{2 t}
$$

and the function defined in (25) is

$$
g_{3}(t)=15+\frac{1-e^{t}}{2 t}-\frac{1-e^{-t}}{t}
$$

Then $g_{3}(t)=0$ for $t \in \mathbb{R}^{-}$if and only if $t \approx-4.136 \ldots$ and, therefore the system (28) has one limit cycle passing thorough $y^{+}(-4.136 \ldots) \approx-7.441 \ldots<y_{m}$.

Now applying the rescaling of time $\tau=-t$ and the change of coordinates $u=x$ and $v=y-5$, we rewrite the system (22) as

$$
\dot{z}= \begin{cases}\left(-u-v+\frac{9}{2},-v+5\right) & \text { for } u>0, \\ \left(-u-v,-v+\frac{\pi^{2}}{\ln (2)^{2}} u\right) & \text { for } u<0,\end{cases}
$$

whose boundary stable focus is $(0,0)$ and its virtual improper stable node is $(-1 / 2,5)$. 


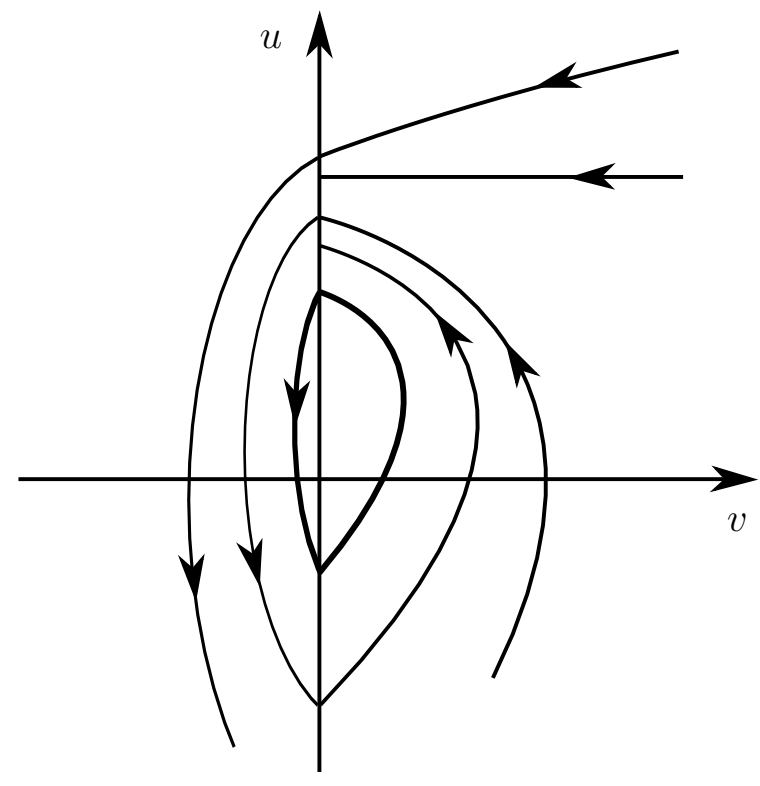

Figure 9. The phase portrait of the piecewise MY-system (29).

Notice that the eigenvalues of (29) have negative real parts. We conclude that system (29) is a piecewise $M Y$-system and it has one limit cycle passing through point $(u, v)=(0,-2.441 \ldots)$, see Figure 9 .

\section{ACKNOWLEDGEMENTS}

The first author is partially supported by a MINECO-FEDER grant MTM201677278-P, a MINECO grant MTM2013-40998-P, and an AGAUR grant number 2014SGR-568. The second author is partially supported by a CAPES PDSE88881.133794/2016-01 and a Ph.D. CAPES grant.

\section{REFERENCES}

[1] J. Bernat, J. Llibre, Counterexample to Kalman and Markus-Yamabe Conjectures in dimension larger then 3, Dynam. Contin. Discrete Impuls. Systems 2 (1996),337-379.

[2] A. Cima, A. van den Essen, A. Gasull, E. Hubbers, F. Mañosas, A polynomial counterexample to the Markus-Yamabe Conjecture, Adv. Math. 131 (1997), 453-457.

[3] R. FeBler, A proof of the two-dimensional Markus-Yamabe stability Conjecture and a generalization, Ann. Polon. Mathematici 62 (1995), 45-75.

[4] A. F. Filippov, Differential equations with discontinuous righthand sides. Mathematics and its Applications (Soviet Series), Kluwer Academic Publishers Group, Dordrecht, 1988.

[5] A. A. Glutsyuk, The complete solution of the Jacobian problem for vector fields on the plane, Comm. Moscow Math. Soc., Russian Math. Surveys 49 (1994), 185-186. 
[6] C. Gutierrez, A solution to the bidimensional Global Asymptotic Stability Conjecture, Ann. Inst. H. Poincaré Anal. Non Linéaire 12 (1995), 627-671.

[7] J. Llibre, D. D. Novaes, M. A. Teixeira, Maximum number of limit cycles for certain piecewise linear dynamical systems, Nonlinear Dyn. 82 (2015), 1159-1175.

[8] L. Markus, H. Yamabe, Global Stability Criteria for Differential Systems, Osaka Math J. 12 (1960), 305-317.

[9] G. H. Meisters, Cz. Olech, Solution of the Global Asymptotic Stability Jacobian Conjecture for the Polynomial Case, Analyse mathèmatiques et applications. Contributions a l'honneur de Jacques-Louis Lions, Gauthier-Villars, Paris 1988, 373-381.

${ }^{1}$ Departament de Matematiques, Universitat Autònoma de Barcelona, 08193 Bellaterra, Barcelona, Catalonia, Spain

E-mail address: jllibre@mat.uab.cat

2 Instituto de Matemática e Estatística, Universidade Federal de Goiás, 74690-900, Goî̂nia, GO, BrazIL

E-mail address: lucyjanedealmeida@gmail.com 